\title{
HETEROGENEIDAD DE LA INMIGRACIÓN INTERNACIONAL RECIENTE EN CHILE. UNA APROXIMACIÓN A TRES GRUPOS NACIONALES A PARTIR DE DATOS DE ENCUESTA
}

\section{HETEROGENEITY OF RECENT INTERNATIONAL IMMIGRATION IN CHILE. AN APPROACH TO THREE NATIONAL GROUPS BASED ON SURVEY DATA}

Dra. Sylvia Soto-Alvarado*,Dr. Fernando Gil-Alonso**, Dra. Isabel Pujadas - Rúbies ${ }^{\star \star *}$

Resumen: La inmigración internacional en Chile ha aumentado progresivamente a partir del año 2001, y a partir del año 2010 se ha diversificado en cuanto al país de origen de los inmigrantes, incrementándose la llegada de personas desde países de Centro y Sur América, entre los que destacan Colombia, Haití y Venezuela. El artículo tiene como objetivo analizar aspectos demográficos, así como la situación socioeconómica y laboral de la población de estos tres países en comparación con la población chilena y con la población inmigrante total. Al declararse inválidos los resultados del Censo chileno de 2012, se ha utilizado en este estudio los microdatos de la encuesta de Caracterización Socioeconómica (CASEN) 2015, cuya muestra permite obtener datos sobre inmigrantes. Los resultados muestran la diversidad que existe entre las poblaciones inmigrantes de los tres países analizados, y el riesgo de exclusión social en la que se encuentran algunas poblaciones extranjeras en Chile.

* Profesora a tiempo parcial, Escuela de Administración Pública, Universidad Austral de Chile, Sede Puerto Montt, Sylvia.soto@uach.cl

** Profesor Agregado, Departamento de Geografía, Universitat de Barcelona, fgil@ub.edu

*** Catedrática de Geografía Humana, Departamento de Geografía, Universitat de Barcelona, ipujadas@ub.edu 
Palabras claves: Migración internacional; migración sur-sur; Chile; exclusión social; participación laboral.

Abstract: Since 2001, international immigration has progressively increased in Chile, and since 2010 the country of origin of immigrants has diversified. There has mainly been an increase in the number of people migrating from South and Central America, and from Colombia, Haiti, and Venezuela particularly. Using 2015 Socioeconomic Characterization (CASEN) survey micro data, this paper analyzes the demographic, socioeconomic and labor conditions of the population of these three countries in comparison to the Chilean and total immigrant populations. Results show that immigrant populations are extremely diverse, and that some foreign populations in Chile are under a great risk of social exclusion.

Key words: International migration; South-South migration; Chile; social exclusion; labor participation.

\section{INTRODUCCIÓN}

La migración Sur-Sur en América Latina se ha incrementado en los últimos años, y esto se ha visto reflejado en los resultados de la ronda de censos de 2010 de los países latinoamericanos, que muestran la intensificación de la migración intrarregional como producto de la globalización, las desigualdades internas y las crisis económicas y políticas de algunos países de América (Martínez, Cano y Soffia, 2014; Martínez y Orrego, 2016).

A pesar de su relevancia numérica (Giorguli y Angoa, 2016) se ha prestado poca atención a las migraciones latinoamericanas intrarregionales que han aumentado considerablemente durante las últimas décadas con la aparición de claros subsistemas migratorios y una mayor diversificación en cuanto lugares de origen, destinos y perfiles migratorios (Cerrutti y Parrado, 2015).

En este contexto y en concordancia con las tendencias de América Latina, la dinámica de la inmigración internacional en Chile también se ha transformado, lo que ha generado un cambio en los patrones tradicionales de la migración internacional en el país. Un reporte del Observatorio Iberoamericano sobre Movilidad Humana (2016) estimó que habría 411.000 extranjeros viviendo en Chile en 
el año 2014 (lo que correspondía a un 2,3\% de la población nacional), tres años más tarde, los resultados del Censo 2017 señalan que residen 746.465 extranjeros en el país, representando un 4,35 de la población total. Estos números muestran un aumento importante de la inmigración reciente, y a ello se ha de sumar la diversificación del origen de los inmigrantes, lo que significa un incremento de la complejidad del fenómeno migratorio en Chile. Por ejemplo, en el Censo del 2002 se constataba un predominio de la inmigración desde países de América del Sur, puesto que en su conjunto componían un $67,85 \%$ de la inmigración total, pero los flujos más cuantiosos provenían de países fronterizos: Argentina (26,12\%), Perú (20,52\%) y Bolivia (5,92\%) (Martínez, 2004; Martínez, 2005; Stefoni, 2005).

Después del Censo de 2002, la tendencia de la inmigración desde países fronterizos ha continuado, pero con algunas variaciones. Mientras que ha disminuido la intensidad del flujo migratorio desde Argentina, el procedente de Bolivia se ha mantenido estable, aumentando su población residente en Chile, y, sorprendentemente, el flujo de inmigrantes desde Perú se ha intensificado, triplicando su población residente en Chile en un periodo de 10 años. Sin embargo, el rasgo novedoso es que a partir del año 2008 se produce un aumento en la llegada de inmigrantes provenientes de otros países de América del Sur y el Caribe que, hasta ese momento, habían tenido una migración muy escasa hacia Chile. Así, en 2015 las siete principales comunidades en Chile son Perú $(33,6 \%)$, Colombia (13,8\%), Argentina $(11 \%)$, Bolivia $(9,4 \%)$, R. Bolivariana de Venezuela (5\%), Ecuador (5\%) y Haití (3,5\%) (Díaz, 2017).

Esta heterogeneidad del colectivo inmigrante en función de su origen geográfico tiene sin duda implicaciones desde el punto de vista socioeconómico que merecen ser investigadas. Por ejemplo, el informe Coyuntura Laboral en América Latina y el Caribe. La inmigración laboral en América Latina (NACIONES UNIDAS - OIT, 2017) señala que Chile es uno de los países latinoamericanos con una proporción más alta de inmigrantes con más de 10 años de escolaridad; sin embargo, la población inmigrante en el país es diversa en este y otros aspectos socioeconómicos, como su integración en el mercado laboral, su grado de cobertura por el sistema de protección social y, en consecuencia, su riesgo de pobreza y exclusión social, dependiendo del país de origen. Por ello, el objetivo de este estudio es indagar sobre las características demográficas, socioeconómicas y laborales de la población de origen colombiano, haitiano y venezolano que 
reside en Chile, realizando una comparación con la población chilena y con el conjunto de la población inmigrante, lo que permitirá observar el grado de vulnerabilidad diferencial entre los grupos de inmigrantes. Se han seleccionado estos tres grupos debido a que son poblaciones que durante la década del 2000 tenían escasa representación en el país y que, sin embargo, han experimentado un crecimiento muy significativo en los últimos años. Particularmente interesante es el caso de la población haitiana: aunque no tiene tanto peso en relación a las demás poblaciones de inmigrantes, la importancia que adquiere en el análisis se debe a que en el Censo de 2002 era un colectivo casi inexistente en el país, y que en los últimos 5 años ha aumentado su stock en más de un $1.000 \%$.

En el momento de iniciarse esta investigación (2015) no existían datos censales actualizados debido a la anulación del Censo de $2012^{1}$ y a que aún no se encontraban disponibles los microdatos del Censo abreviado realizado el año $2017^{2}$. Ello nos hizo preguntarnos sobre la posibilidad de utilizar fuentes estadísticas alternativas que nos permitieran estudiar en profundidad estos cambios en la dinámica migratoria del país así como las características de las nuevas poblaciones de extranjeros. Así, nos planteamos utilizar las cifras del registro del Departamento de Extranjería y Migración y los datos de la encuesta de Caracterización Socioeconómica (CASEN), de

1 Tras un gran debate técnico y político sobre la calidad de los resultados del Censo de 2012, una auditoría técnica liderada por el Instituto Nacional de Estadística de Chile, con asesoría directa del Centro Latinoamericano y Caribeño de Demografía (CELADE) de la CEPAL concluyó en 2014 que «los datos generados para el año 2012 no cumplen con los estándares para que este operativo sea denominado como un censo» (INE, 2014)

2 En junio del 2018 se han liberado los microdatos del Censo de 2017, y los resultados de éste se han comparado con los obtenidos a partir de la encuesta CASEN en la tesis doctoral de Sylvia Soto titulada "La Inmigración internacional reciente en Chile. El proceso migratorio en la región de Los Lagos”, dirigida por los doctores Isabel Pujadas Rúbies y Fernando Gil Alonso, que fue defendida en noviembre del 2018 en la Universidad de Barcelona. Los datos censales de 2017 verifican los principales resultados obtenidos a partir de la encuesta CASEN, si bien actualizan y añaden exactitud a ciertas cifras presentadas en este artículo. Por ejemplo, los colectivos colombiano, venezolano y haitiano son los que han tenido mayor crecimiento en el periodo intercensal, y especialmente a partir del año 2010. Así también se verifica la fuerte masculinización de la migración haitiana y la tendencia a la masculinización de la población extranjera total en el país. Igualmente, el nivel de escolaridad de los colectivos analizados coincide con los resultados de la encuesta CASEN. 
manera similar a como Gil-Alonso hizo en España con el registro de tarjetas de residencia temporales expedidas por el Ministerio del Interior (Gil-Alonso, 2010) y con la Encuesta de Población Activa - EPA (Gil-Alonso y Domingo, 2008; Gil-Alonso y Vidal, 2015). Finalmente se han utilizado los microdatos de la encuesta CASEN de 2015 , la cual se realiza cada dos años y tiene por objetivo principal medir la situación socioeconómica de los hogares y de la población. Pese a las limitaciones propias de este instrumento para los estudios sobre migración internacional — pues su muestra no está diseñada para captar este fenómeno-, se consideró que, dado el importante número de población encuestada y siempre que se tuviera en cuenta en el análisis el sesgo estadístico existente, podría ser un aporte válido en los estudios y caracterizaciones sobre la población extranjera, tanto por la riqueza de las variables contenidas en el cuestionario como por proporcionar una valiosa información sobre estos colectivos en los periodos entre censos. Creemos que los resultados obtenidos permiten avalar la validez de la encuesta CASEN para realizar una primera aproximación al fenómeno migratorio.

Si la utilización de esta fuente es la principal aportación del artículo a los estudios sobre la migración internacional en Chile, los resultados también creemos que son interesantes, pues validan la hipótesis de partida: los desiguales niveles de riesgo de exclusión social entre los tres colectivos analizados - y, en general, entre las diferentes nacionalidades- derivan de una inserción desigual en el mercado de trabajo chileno, que a su vez está condicionada tanto por las características socioeconómicas de partida de los grupos analizados (por ejemplo, el nivel educativo) como por la mirada que sobre los distintos grupos de inmigrantes tiene la población chilena, con sus derivadas de racismo y discriminación. Los fundamentos teóricos que justifican esta hipótesis se explican en el siguiente apartado, al que sigue una descripción de las fuentes y los métodos utilizados en el análisis. El mayor número de páginas se dedica a la descripción de los resultados, cerrando el artículo la discusión de éstos y las conclusiones.

\section{REVISIÓN DE LITERATURA}

La migración internacional laboral es explicada por las teoría clásica y neoclásica como producto de las desigualdades de empleo y salarios entre países, donde operan factores de expulsión y 
atracción entre unos y otros. Así, en los países de origen de la población migrante predominan factores negativos en cuanto a empleo y salario y en los países receptores predominan factores positivos. El análisis macro sobre los factores que determinan la migración es complementado por la teoría neoclásica añadiendo una dimensión micro relativa a los factores racionales que inciden en la decisión de migrar, como los costos tangibles e intangibles de la migración y el cálculo del costo-beneficio (Massey y otros, 1993; Arango, 2003). Esta perspectiva tradicional para la explicación de las migraciones, aunque aborda aspectos importantes en la decisión de migrar, necesariamente debe ser complementada con otras perspectivas teóricas, puesto que por sí sola no logra dar cuenta de la complejidad de las actuales migraciones internacionales, como que siga existiendo migración hacia un destino, aunque las diferencias salariales con el lugar de origen hayan desaparecido, o como la existencia de migración de retorno (Hass, 2011).

Por otro lado, la migración internacional no se debe exclusivamente a las desigualdades salariales entre países, sino que pueden existir otros factores económicos como la desigualdad interna de los países o la percepción de deprivación relativa, entre otros, que pueden ser los determinantes en la decisión de migrar, motivos que se encuentran presentes en los países de América Latina y el Caribe. "Mientras más desigual sea la distribución de ingresos en una comunidad determinada, más se sentirá la privación relativa y mayores serán los incentivos para la emigración" (Arango, 2003, p. 12).

Sin embargo, como ya se ha mencionado, la migración en la región no es exclusivamente económica; dependiendo de los países de origen y los países de destino, ésta puede ser por motivos políticos o de inseguridad. "En el caso de los colombianos, además se suman los motivos vinculados al conflicto armado y a la inseguridad. En este caso, la complejidad de estos en el contexto de origen dificulta enormemente la identificación de causas excluyentes que impulsan la migración, lo que permite hablar de flujos mixtos de colombianos..." (Martínez (Ed.), 2011, p. 128). Situación similar sucede actualmente con la migración de origen venezolano, que ha aumentado mucho los últimos años como consecuencia del conflicto político interno existente en el país y que tiene un origen mixto también, en el que intervienen, principalmente, causas económicas y de seguridad (Páez, 2015). 
En la última década la migración intrarregional en América Latina y el Caribe se ha intensificado, y aunque persisten patrones de emigración hacia el exterior junto con inmigración de ultramar, sin duda son los intercambios regionales los que han cobrado mayor fuerza. Los destinos tradicionales de la emigración latinoamericana han sido Estados Unidos y España, y, aunque continúan vigentes, han venido experimentando una tendencia regresiva que se suele explicar por el impacto negativo que tuvo la crisis del año 2008 sobre los mercados laborales y los salarios de ambos países (Martínez y otros, 2014; Martínez y Orrego, 2016). En este contexto, la migración en la región se ha diversificado en cuanto a los movimientos intrarregionales, en tanto países que tenían destinos emigratorios definidos los han ampliado a otros países de la misma región y, a su vez, países que se habían caracterizado por recibir preferentemente migración fronteriza se han transformado en destinos emergentes dentro de la región, como es el caso de Chile. Así, la dinámica de la migración internacional en Latinoamérica es distinta a la que se puede observar en países desarrollados que reciben población migrante de todos los continentes, puesto que en los países latinoamericanos la migración ha adquirido un carácter regional y no global (Czaika y Hass, 2015).

Un factor que ha contribuido a la intensificación de la migración intrarregional es el político, el rol que juegan los Estados en la definición de políticas migratorias que favorecen o no el movimiento de población y los tratados y acuerdos internacionales en materia de comercio y trabajo (Arango, 2003). En el caso latinoamericano se sostiene que, durante la década de los noventa, la apertura de los mercados y la globalización, acompañada de la liberalización de los controles de tránsito para el comercio, el turismo y el trabajo, habría favorecido la migración intrarregional (Duran y Massey, 2010). Un ejemplo de esto es el acuerdo sobre residencia de los estados parte del MERCOSUR, Bolivia y Chile.

En cuanto a las características que tiene la migración en la región, una de ellas es la marcada participación de las mujeres (Martínez (Ed.), 2008). La feminización de las migraciones intrarregionales no es nueva, sin embargo, hay algunos países de origen en los que la migración femenina es más marcada que en otros, como la migración dominicana y peruana. También hay países de destino que atraen más mujeres, lo que obedece a la demanda de determinados sectores de actividad y ocupación. Este no es un fenómeno 
exclusivo de la migración intrarregional, pues también la inmigración latinoamericana en España se ha caracterizado por la marcada feminización de algunos de sus colectivos nacionales; ello se ha explicado por la demanda de trabajo femenino en sectores como el cuidado de infantes y personas mayores, el servicio doméstico y en otros de baja calificación, sectores en los que las mujeres latinoamericanas habría sustituido a las españolas al incorporarse éstas a puestos de trabajo mejor calificados y remunerados (Gil-Alonso y Domingo, 2008). Sin embargo, a partir del 2010, se ha observado que, aunque la migración interregional sigue siendo mayoritariamente femenina en términos generales, hay algunos colectivos, en particular el haitiano, que presenta un marcado sesgo masculino (Martínez y Orrego, 2016).

Respecto al grado de calificación de los migrantes intrarregionales, en oposición a la idea de que los migrantes calificados se desplazaban hacia destinos fuera de la región y los migrantes de baja calificación se movían dentro de la región, los datos disponibles demuestran que la migración intrarregional presenta tanto migración calificada como de baja calificación. Duran y Massey (2010) señalan que la distinción entre migrantes calificados y de baja calificación es necesaria porque los dos tipos de migrantes raramente interactúan, aun cuando sean del mismo país. Los migrantes profesionales habitualmente se localizan en las ciudades capitales y viajan en busca de oportunidades para educación, trabajo o desarrollo profesional. Por otro lado, para los migrantes calificados, la teoría señala que, si bien el desplazamiento tiene dentro de sus objetivos encontrar empleos afines al nivel educacional que poseen y así maximizar sus ganancias, la elección del destino está sujeta también a las restricciones migratorias de cada país; por ello se optará por el destino que ofrezca facilidades legales para residir (Allen y Fazito, 2017). En este sentido, los migrantes calificados buscan no correr riesgos en el lugar de destino, en cuanto a la posibilidad de tener una residencia legal.

Chile no representa una excepción regional en el hecho de recibir tanto migración calificada como de baja calificación, sin embargo, destaca respecto a otros países de su entorno en el mayor peso relativo - no absoluto- de la migración calificada (Martínez, 2008). Las particularidades de la emigración con destino a este país se explican muy bien gracias a la teoría del "mercado de trabajo dual" propuesta por Piore (1979), pues ésta aporta una explicación desde la sociedad receptora de la migración. Esta teoría explicaría 
la existencia en Chile, como país semiperiférico en vías de desarrollo, de un mercado de trabajo segmentado o dual debido a "la división de la economía en un sector primario, intensivo en capital, y un sector secundario, intensivo en trabajo y de baja productividad" (Arango, 2003, p. 14). De acuerdo con este marco teórico, la mayoría de los inmigrantes extranjeros se insertarían principalmente en el sector secundario, que tienden a tener condiciones de trabajo más desventajosas para sus empleados. Esto porque "los bajos salarios, las condiciones inestables y la carencia de expectativas sobre movilidad en el sector secundario hace difícil atraer trabajadores nativos" (Massey et al. 2000, p. 19). Simultáneamente, y gracias al nivel de desarrollo de Chile en comparación con otros países de su región, también existiría una demanda de trabajadores foráneos altamente cualificados que encontrarían trabajo en el mercado de trabajo primario de la economía chilena. Así, esta teoría aporta un marco conceptual adecuado para analizar las diferentes características de la inserción en el mercado laboral chileno de los inmigrantes de las nacionalidades analizadas, pues como se verá más adelante, existe una cierta correlación entre el origen nacional de los inmigrantes y sus características en cuanto al nivel de calificación.

No obstante, cabe mencionar que las características sociodemográficas de los migrantes son un elemento que incide directamente en la incorporación en la sociedad de destino, y en las condiciones laborales y sociales que tendrán, aumentando la vulnerabilidad de algunas poblaciones sobre otras, pero no es el único factor que la explica. Otros factores, como la desigual discriminación que sufren en Chile los diferentes grupos de inmigrantes en función de la construcción de jerarquías racializadas en la sociedad de acogida, que perjudican más a aquellos colectivos con fenotipos menos europeos (Tijoux, 2016; Tijoux y Córdova, 2015) también podrían ayudar a explicar el mayor riesgo de exclusión social en algunas nacionalidades como bolivianos, peruanos, colombianos y, especialmente, haitianos, que además sufren una barrera lingüística al no ser castellanohablantes. Así, el racismo se expresa además en la jerarquización de clases y en la racialización de la mano de obra, en la medida que se asocian ciertos nichos laborales a determinadas nacionalidades $\mathrm{o}$ a inmigrantes afrodescendientes (Rojas et al., 2015). En este sentido, el racismo produce mecanismos de inclusión y exclusión de la población extranjera residente en Chile, que además de expresarse socialmente se expresa en las condiciones laborales de extranjeros, 
produciéndose lo que Valenzuela et al., (2014) han denominado "inclusión diferencial”, donde existe una integración al mundo laboral, pero en condiciones de mayor precariedad ante los autóctonos.

\section{METODOLOGÍA Y FUENTES}

Este estudio es un análisis estadístico de datos secundarios de la encuesta CASEN (Caracterización Socioeconómica Nacional) del año 2015. La encuesta se realiza cada dos años y tiene por objetivo principal la medición del nivel socioeconómico de los hogares y la población nacional, sin embargo, también mide otras dimensiones como salud, participación social y migración, entre otras. La responsabilidad de su realización es del Ministerio de Desarrollo Social de Chile, y el año 2015 la Universidad de Chile estuvo a cargo del levantamiento y procesamiento de los datos.

La encuesta CASEN 2015 es representativa a nivel nacional, regional, por zona de residencia (urbana y rural) y para 139 comunas que, sumadas, concentran el $80 \%$ o más de las viviendas de cada región. La muestra objetiva fue de 82.370 viviendas y 266.968 personas encuestadas.

Así, la muestra obtenida es la siguiente:

\begin{tabular}{|c|c|c|}
\hline & Muestra & $\begin{array}{c}\text { Población estimada (datos } \\
\text { ponderados) }\end{array}$ \\
\hline Muestra total & 266.968 & 17.552 .505 \\
\hline Extranjeros & 4.851 & 465.319 \\
\hline Colombianos & 571 & 63.481 \\
\hline Venezolanos & 149 & 20.816 \\
\hline Haitianos & 111 & 15.705 \\
\hline
\end{tabular}

El muestreo de la encuesta CASEN no considera a los migrantes en su definición, por tanto, puede producirse un sesgo estadístico en el que esta población puede quedar subrepresentada. Sin embargo, "dada la dificultad de contar con una fuente que permita cuantificar y proyectar la dinámica migratoria del país, esta constituye una buena alternativa para esbozar tendencias, por la semejanza de las preguntas y su representatividad a nivel nacional" (Díaz, 2017, p. 237). Por ello, con el fin de reducir el sesgo se han estimado intervalos de 
confianza del 95\% y el error muestral para el total de extranjeros, para cada una de las poblaciones estudiadas y para las variables categóricas utilizadas. En los casos en que el intervalo de confianza es demasiado amplio se ha optado por no colocar el dato.

Además de los datos demográficos y socioeconómicos tradicionales, en el artículo se utiliza la variable de pobreza, siendo necesaria una aclaración sobre cómo la encuesta CASEN mide esta variable. La encuesta incorpora 3 formas de medir la pobreza, de las que se han seleccionado dos para ser presentada en los resultados, y que son las que siguen:

- Pobreza por ingreso: El cálculo que se realiza es de acuerdo con la Metodología de Medición de Ingresos y de la Pobreza por Ingresos, publicada en enero 2015 por la CEPAL, en la cual se considera el ingreso disponible del hogar para su medición y si este cumple con un estándar mínimo de ingreso necesario para satisfacer un conjunto de necesidades básicas.

- Pobreza multidimensional, de 5 dimensiones: Considera 5 dimensiones y 3 indicadores para cada dimensión. Las dimensiones son: educación (indicadores: asistencia, rezago escolar, escolaridad), salud (indicadores: malnutrición en niños/as, adscripción al sistema de salud, atención), trabajo y seguridad social (indicadores: ocupación, seguridad social y jubilaciones), vivienda y entorno (indicadores: habitabilidad, servicios básicos, entorno), y redes y cohesión social (indicadores: apoyo y participación social, trato igualitario y seguridad). Las primeras cuatro dimensiones se ponderan en $22,5 \%$, y última en un $10 \%$. Se considera un hogar en situación de pobreza si presenta un $22,5 \%$ o más de carencias.

\section{RESULTADOS}

\subsection{Aspectos demográficos de la población haitiana, colombiana y venezolana}

Una de las principales características de las poblaciones analizadas es el crecimiento que han tenido en Chile entre 2002 y 2015 (ver cuadro 1) y cómo ha aumentado el peso relativo que estas tienen en relación al total de extranjeros residentes en el país (ver cuadro 2). 
Cunaro 1

POBLACIÓN EXTRANJERA EN CHILE, TOTAL Y PAÍSES ANALIZADOS. 2002-2015

\begin{tabular}{|l|c|c|c|}
\hline Países & Censo 2002 & Censo 2012 & Casen 2015 (95\% IC) \\
\hline Colombia & 4.095 & 27.411 & $63.481(38.923-88.039)$ \\
\hline Venezuela & 4.338 & 7.897 & $20.816(7.923-33.708)$ \\
\hline Haití & 50 & 1.675 & $15.705(6.737-24.673)$ \\
\hline Total extranjeros & 184.464 & 332.288 & $465.319(414.661-515.977)$ \\
\hline
\end{tabular}

Fuente: elaboración propia con microdatos INE (2017) y Ministerio Desarrollo Social (2015).

Para el Censo del 2002 los colectivos colombianos y venezolano tenían un peso relativo de poco más del $2 \%$, respectivamente, frente al total de extranjeros, mientras que en el año 2015, según los resultados de la encuesta CASEN, esta proporción se ha sextuplicado en el caso de los colombianos - pasando a ser el segundo colectivo más numeroso del país - y se ha duplicado en el caso de los venezolanos (ver cuadro 2).

\section{CuAdro 2}

PROPORCIÓN DE POBLACIÓN EXTRANJERA PROCEDENTE DE LOS PAÍSES ANALIZADOS. 2002-2015

(Porcentajes)

\begin{tabular}{|l|c|c|}
\hline Países & Censo 2002 & Casen 2015 (95\% IC) \\
\hline Colombia & 2,22 & $13,60(12,0-15,5)$ \\
\hline Venezuela & 2,35 & $4,50(3,4-5,9)$ \\
\hline Haití & 0,02 & $3,40(2,7-4,2)$ \\
\hline
\end{tabular}

Fuente: elaboración propia con microdatos INE (2017) y Ministerio Desarrollo Social (2015).

3 Se utilizan los datos del Censo 2012 únicamente como valores referenciales en comparación con los datos aportados por la encuesta Casen, pues ha de tenerse en cuenta que el Censo fue invalidado como información de uso oficial debido a los errores en su realización. 
Especial es el caso de haitianos, que en el año 2002 no alcanzaban a representar el 0,1 por ciento de la población extranjera y en 2015 han llegado a representar al 3,4 por ciento del total de la población extranjera, situándose como el séptimo colectivo más numeroso en Chile (Díaz, 2017).

El aumento de estas poblaciones que se ha señalado no ha sido paulatino desde el año 2002, sino que la mayor parte de estas poblaciones han llegado al país en los últimos 5 años, es decir, a partir del año 2010 (ver cuadro 3). Esta cifra es muy alta en el colectivo haitiano, un $88 \%$ ha llegado a partir del 2010 , y en el caso de venezolanos y colombianos un $71 \%$ y un $69 \%$, respectivamente, han llegado desde esta fecha. Por el contrario, el $40 \%$ de la población extranjera total ha llegado desde el 2010. Estos datos indican lo reciente de la migración de las tres poblaciones seleccionadas, y también del aumento de la intensidad de la inmigración internacional en Chile.

\section{CuAdro 3}

\section{PRINCIPALES INDICADORES DEMOGRÁFICOS DE LOS COLECTIVOS EXTRANJEROS SELECCIONADOS CASEN 2015}

(Distribución porcentual)

\begin{tabular}{|l|c|c|c|c|}
\hline & Total extranjeros & Colombia & Venezuela & Haití \\
\hline $\begin{array}{l}\text { \% Población } \\
\text { Ilegada a partir } \\
\text { del } 2010\end{array}$ & $40,4(34,7-46,4)$ & $68,7(52,0-81,7)$ & $70,5(48,4-85,9)$ & $87,7(70,2-95,6)$ \\
\hline$\%$ Zona Urbana & $97,1(96,1-97,9)$ & $98,5(96,6-99,3)$ & $98,2(92,11-99,61)$ & $99,2(94,4-100)$ \\
\hline $\begin{array}{l}\text { \% Región } \\
\text { metropolitana }\end{array}$ & $69,1(65,1-72,8)$ & $73,7(60,7-83,5)$ & $86,0(71,9-93,7)$ & $99,8(99-100,0)$ \\
\hline $\begin{array}{l}\text { \% Menores de } \\
15 \text { años }\end{array}$ & $12,9(11,2-15,0)$ & $15,0(9,9-22,1)$ & $10,3(4,5-21,7)$ & $16,1(9,0-27,3)$ \\
\hline$\%$ 15 a 64 años & $83,3(81,3-85,2)$ & $83,8(78,3-88)$, & $87(77,9-92,8)$ & $82,1(71,1-89,6)$ \\
\hline$\% 65$ años 0 más & $3,7(3,1-4,5)$ & $1,2(0,5-3,2)$ & $2,7(0,9-7,8)$ & $1,8(0,4-6,7)$ \\
\hline $\begin{array}{l}\text { Índice de } \\
\text { Masculinidad }\end{array}$ & $0,93(0,89-0,95)$ & $0,94(0,89-0,96)$ & $0,81(0,63-0,86)$ & $1,51(1,42-1,54)$ \\
\hline
\end{tabular}

Fuente: elaboración propia con microdatos encuesta Casen 2015. 
El cuadro 3 también muestra que un 69\% de la población extranjera en Chile reside en la región metropolitana, donde se encuentra la capital del país y se concentra además el $40 \%$ de la población nacional. Los colectivos estudiados presentan una concentración mayor en la región metropolitana (colombianos, 74\%; venezolanos, 86\%; y haitianos, 99\%) que el conjunto de los extranjeros, y la tendencia de estas poblaciones es residir en algunas comunas específicas de la región. Así, el 56\% de los haitianos residen en la comuna de Quilicura, una comuna periférica del Gran Santiago de aproximadamente 210.000 habitantes, por lo que alrededor del $5 \%$ de la población es de origen haitiano. Por su parte, un $45 \%$ de la población colombiana y el $50 \%$ de la población venezolana se concentran mayoritariamente en la comuna de Santiago que tiene una población aproximada de 500.000 habitantes. Esta diferencia entre la población total y las poblaciones estudiadas puede deberse, por una parte, a que algunas poblaciones de países limítrofes tienen dinámicas de migración fronteriza, como el colectivo boliviano, que se concentra en las regiones del Norte Grande, y el colectivo argentino, que se encuentra presente en todas las regiones del país.

La población venezolana es más feminizada que el total de extranjeros, mientras que la población colombiana tiene una composición de hombres y mujeres similar al del total de extranjeros residentes en el país. Estas cifras se encuentran en concordancia con la tendencia regional de migración feminizada (Martínez, Cano y Soffia, 2014). Por el contrario, la población haitiana en Chile es masculinizada, siendo esta la tendencia de los haitianos que migran hacia países latinoamericanos (Martínez y Orrego, 2016).

La población de los tres colectivos son en su mayoría población entre 15 y 64 años, económicamente activa, con escasa población adulta mayor e infantil. Esta estructura etaria muestra el inicio reciente de los flujos migratorios desde estos países hacia Chile — pues a estos colectivos no les ha dado tiempo de reagrupar a su descendencia, si la tienen, ni de envejecer en el país de destino-, y del carácter eminentemente laboral de las migraciones. Para evitar diferencias de estructura etaria entre la población extranjera y la chilena, los próximos análisis se realizan comparando la población en edad activa, esto es, entre 15 y 59 años, o entre 18 y 64 años, dependiendo de las variables analizadas. 


\subsection{Situación socioeconómica de la población analizada}

El nivel educacional de las poblaciones analizadas es muy diverso entre ellas (Gráfico 1). Mientras que en el colectivo venezolano alrededor del $80 \%$ tiene estudios superiores, lo que lo sitúa como migrantes calificados, por el contrario más del $90 \%$ del colectivo haitiano tiene estudios primarios y secundarios, con menos de un $10 \%$ de su población con estudios superiores. Los colombianos muestran un nivel educacional diverso en el que predominan personas con educación secundaria completa, aunque la proporción que tiene educación superior es mayor que la de la población chilena. La distribución relativa del nivel educacional de colombianos es similar al de la población extranjera total, mostrando también este último grupo una proporción de población calificada mayor que la población chilena calificada. La diversidad de la población colombiana en cuanto a nivel educacional, estrato social y raza incide en la integración social diferenciada de las personas del mismo colectivo (GissiBarbieri y Ghio-Suarez, 2017).

\section{GRÁFICO 1}

\section{NIVEL EDUCACIONAL POBLACIÓN CHILENA Y EXTRANJERA ENTRE 18 Y 59 AÑOS}

(Distribución porcentual)

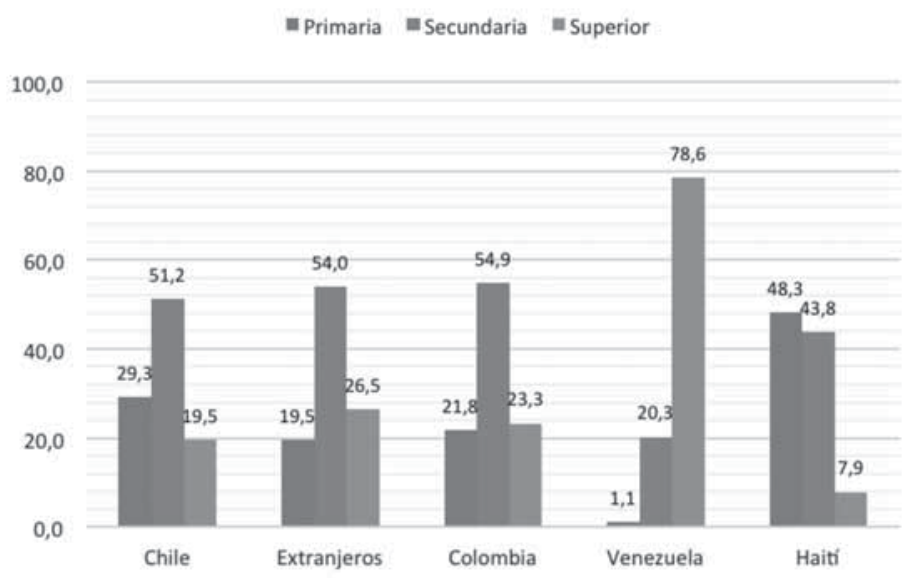

Fuente: elaboración propia con microdatos Encuesta Casen 2015. 
Respecto a la situación de pobreza por ingreso, más del 10\% del colectivo haitiano se encuentra en situación de pobreza extrema, es decir, sus ingresos mensuales son inferiores a 152,74 dólares ${ }^{4}$. Esta proporción constituye una alarma sobre las condiciones de vida de este colectivo en Chile y constituye una expresión de las brechas raciales y de capacidades de esta población. Por su parte, la población venezolana y colombiana que se encuentra en situación de pobreza por ingreso es menor que la chilena y que el total de extranjeros, aun considerando el intervalo de confianza (ver cuadro 4).

\section{CUADRo 4}

INDICADORES DE SITUACIÓN SOCIECONÓMICA DE LA POBLACIÓN CHILENA Y EXTRANJERA ENTRE 15 Y 64 AÑOS

(Distribución porcentual)

\begin{tabular}{|c|c|c|c|c|c|}
\hline & \multicolumn{5}{|c|}{ Estimación con 95\% IC (Inferior - Superior) } \\
\hline & Chile & $\begin{array}{c}\text { Total } \\
\text { extranjeros }\end{array}$ & Colombia & Venezuela & Haití \\
\hline \multicolumn{6}{|l|}{ Pobreza por ingreso } \\
\hline Pobreza extrema & $3,2(3,1-3,3)$ & $3,5(2,8-4,4)$ & $0,9(0,4-1,9)$ & $0,3(0,1-1,5)$ & $10,9(4,4-24,4)$ \\
\hline Pobreza no extrema & $7,6(7,4-7,8)$ & $5,3(4,5-6,2)$ & $2,7(1,2-5,9)$ & $0,3(0,0-2,4)$ & $2,2(0,5-8,4)$ \\
\hline Sin pobreza & $89,2(89,0-89,4)$ & $91,2(90,0-92,3)$ & $96,4(93,4-98,1)$ & $99,3(97,7-99,8)$ & $86,9(73,9-94,0)$ \\
\hline $\begin{array}{l}\text { Situación de Pobreza } \\
\text { (5 dimensiones) }\end{array}$ & $20,3(20,1-20,6)$ & $21,2(19,1-23,5)$ & $31,4(24,8-38,8)$ & $10,1(5,1-19,0)$ & $41,9(30,6-54,1)$ \\
\hline \multicolumn{6}{|l|}{ Sistema de salud } \\
\hline FONASA (Público) & $75,3(75,0-75,7)$ & $64,0(61,1-66,8)$ & $60,4(52,8-67,4)$ & $43,5(29,1-59,1)$ & $82,4(72,2-89,4)$ \\
\hline ISAPRE (Privado) & $16,6(16,3-16,9)$ & $17,2(15,1-19,5)$ & $11,8(8,5-16,1)$ & $24,6(16,3-35,4)$ & $1,7(0,5-6,0$ \\
\hline No tiene & $3,4(3,3-3,5)$ & $14,5(12,4-16,9)$ & $18,5(13,2-25,3)$ & $24,(12,7-42,5)$ & $14,7(8,3-24,8)$ \\
\hline Otro & $4,6(4,5-4,8)$ & $4,4(3,4-5,6)$ & $9,3(5,6-15,1)$ & $7,7(1,6-26,5)$ & $1,2(0,3-5,0)$ \\
\hline
\end{tabular}

Fuente: elaboración propia con microdatos encuesta Casen 2015.

En la estimación de pobreza multidimensional para los colectivos estudiados, el intervalo de confianza es demasiado amplio, lo

4 Según el costo de la canasta básica de alimentos en agosto del 2015 y calculado en dólares el día 25 de enero del 2017. 
que influye en la veracidad de los resultados. Aun así, considerando el IC, las poblaciones colombiana y haitiana, muestran una proporción de individuos en situación de pobreza mucho más alta que el total de extranjeros y de chilenos. Sobre todo, el colectivo haitiano. Por el contrario, el colectivo venezolano tiene una proporción de población en situación de pobreza multidimensional más baja que la chilena y que las demás poblaciones estudiadas, especialmente en el caso de los hombres venezolanos. El aporte de la medición de pobreza multidimensional es que complementa la tradicional medida de pobreza en función del ingreso y canasta básica, midiendo otras dimensiones relacionadas con vivienda y entorno, redes sociales, salud y trabajo y seguridad; dimensiones que en el caso de los extranjeros muchas veces no se encuentran resueltas, de ahí la elevada proporción de ellos que se encuentran en esta situación, aunque el porcentaje de extranjeros que están en situación de pobreza medida por ingreso sea aparentemente más bajo.

En cuanto al sistema de salud en Chile, es necesario explicar primero cómo funciona. Este es un sistema mixto, es decir público y privado, y las personas pueden optar libremente por escoger uno u otro (siempre que el nivel de ingresos lo permita). Por ello la pertenencia a un sistema u otro es también un indicador de estatus social, además de permitir observar si la población tiene acceso al derecho básico en salud. El sistema público se llama Fondo Nacional de Salud (FONASA), y el sistema privado Instituciones de Salud Previsional (ISAPRES), que agrupa a 13 empresas aseguradoras en salud ${ }^{5}$.

La población haitiana en su mayoría se encuentra adherida al sistema de salud público FONASA. Por su parte, un 60\% del colectivo colombiano y menos del $45 \%$ de la población venezolana pertenece a FONASA. Por otro lado, casi un $25 \%$ de los venezolanos no cuentan con ningún sistema de salud, situación similar sucede con una quinta parte de la población colombiana, y en torno a un 15\% de la haitiana, aunque estas últimas estimaciones presentan un IC demasiado alto. En cualquier caso, el porcentaje de extranjeros sin sistema de salud es mucho más elevado que entre la población chilena, lo que es un indicador de su situación de vulnerabilidad.

5 Se excluye en la presentación el sistema de salud de quienes pertenecen a las Fuerzas Armadas y Carabineros de Chile, que es un sistema de salud exclusivo y no relevante en este análisis. 
GRÁFICO 2

ESTIMACIÓN INGRESO MENSUAL AUTÓNOMO CORREGIDO. IC 95\% (Media)

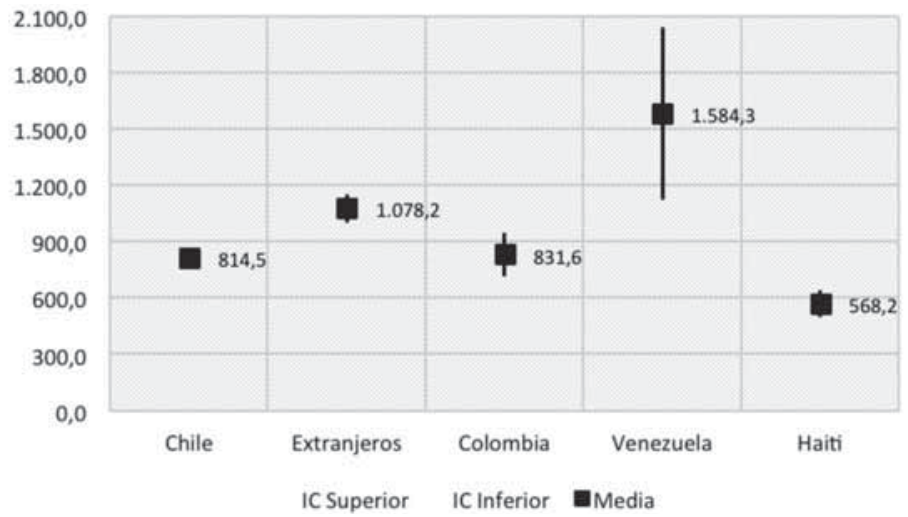

Fuente: elaboración propia con microdatos Ministerio de Desarrollo Social (2017).

* Calculado en dólares al 11/01/2018.

Respecto al nivel de renta de las poblaciones estudiadas, se analizó el promedio de "ingreso autónomo mensual corregido" en cada colectivo. La media del ingreso autónomo del total de extranjeros residentes en Chile es mayor en algo más de 200 dólares mensuales en relación al ingreso medio de la población chilena (que incluye a la población jubilada, menos numerosa entre los extranjeros y casi inexistente entre los tres colectivos analizados), y el ingreso autónomo de colombianos es muy similar al de chilenos (ver Gráfico 2). En los extremos encontramos al colectivo venezolano que presenta una media de ingreso autónomo mensual que prácticamente dobla el ingreso de chilenos - sin embargo, tiene un intervalo de confianza muy amplio-, y en el extremo inferior el colectivo haitiano, que tiene una media de ingresos autónomo mensual inferior en casi 300 dólares al ingreso promedio de chilenos.

\subsection{Inserción de haitianos, colombianos y venezolanos en el mercado laboral}

La tasa de ocupación de la población extranjera en Chile es del $74 \%$, muy por encima de la tasa de ocupación de los chilenos, que es 
del 60\% para la población entre 15 y 64 años, manifestando el marcado carácter de migración laboral que tiene la inmigración en Chile (ver cuadro 5). Exceptuando a los venezolanos, los demás colectivos estudiados trabajan, en promedio, al menos 2 horas más semanales que los chilenos, cumpliendo jornadas que superan las 45 horas semanales. La ley laboral en Chile establece 45 horas laborales semanales, por lo que, principalmente el colectivo haitiano tiene jornadas laborales demasiado extensas.

CuAdro 5

INDICADORES DE OCUPACIÓN Y EMPLEO DE EXTRANJEROS Y CHILENOS, OCUPADOS ENTRE 15 Y 64 AÑOS

(Distribución porcentual)

\begin{tabular}{|l|c|c|c|c|c|}
\hline & Chile & Extranjeros & Colombia & Venezuela & Haití \\
\hline$\%$ Ocupados & $60,1(59,7-60,4)$ & $74,3(71,9-76,6)$ & $74,6(66,9-81,1)$ & $80,5(70,8-87,6)$ & $81,5(70,6-88,9)$ \\
\hline $\begin{array}{l}\text { Asalariados con } \\
\text { contrato escrito }\end{array}$ & $86,5(86,1-86,8)$ & $86,3(84,1-88,3)$ & $85,5(78,4-90,5)$ & $91,4(65,3-98,4)$ & $77,1(61,4-87,7)$ \\
\hline $\begin{array}{l}\text { Horas promedio } \\
\text { trabajo semanal }\end{array}$ & $43,0(42,8-43,1)$ & $44,8(44,0-45,6)$ & $45,2(43,0-47,4)$ & $43,0(39,5-46,3)$ & $48,4(41,9-54,8)$ \\
\hline
\end{tabular}

Fuente: elaboración propia con microdatos Ministerio de Desarrollo Social (2017).

Respecto de la formalidad de los contratos, el porcentaje de asalariados extranjeros con contrato escrito es muy similar al de los chilenos ( $87 \%$ y $86 \%$, respectivamente), lo que sucede también con la población colombiana, mientras que el porcentaje es superior entre los venezolanos. Por su parte, los extranjeros de origen haitiano son el grupo que tiene un mayor porcentaje de asalariados en forma irregular, es decir, sin contrato, lo que muestra de nuevo su mayor vulnerabilidad laboral.

A continuación, se presenta las ocupaciones de los colectivos estudiados, según el nivel de competencias requerido para su desempeño $^{6}$. La OIT agrupa los diez grandes grupos de ocupaciones

6 Nivel de competencias 1: trabajadores no calificados; nivel de competencias 2: empleados de oficina, trabajadores de servicios y vendedores de comercio, agricultores y trabajadores calificados agropecuarios y pesqueros, oficiales, operarios, artesanos de artes mecánicas y de otros oficios y operadores 
de CIUO-08 en cuatro grandes grupos de acuerdo con el nivel de competencias requerido para desarrollar una ocupación, nivel que se mide considerando la naturaleza del trabajo desempeñado, el nivel de enseñanza formal y la cantidad de formación informal en el empleo o experiencia previa ${ }^{7}$.

\section{GRÁFICO 3}

\section{ESTIMACIÓN DEL NIVEL DE COMPETENCIAS DE LAS OCUPACIONES QUE DESARROLLAN CHILENOS Y EXTRANJEROS ENTRE 15 Y 64 AÑOS}

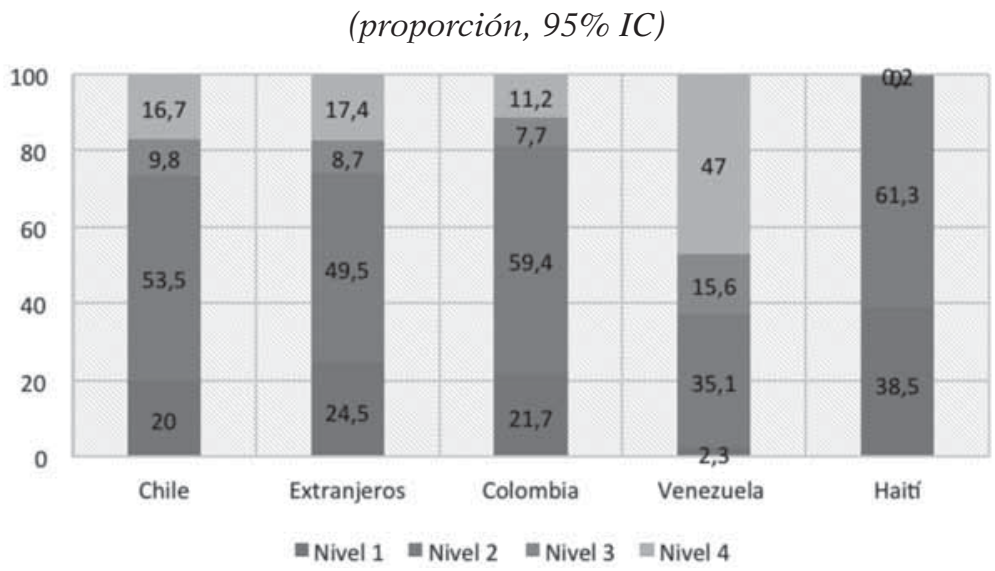

Fuente: elaboración propia con microdatos de Ministerio de Desarrollo Social (2017).

de instalaciones y máquinas y montadores; nivel de competencias 3: Técnicos y profesionales de nivel medio y (ocasionalmente) miembros del poder ejecutivo, legislativo, personal directivo de administración pública y empresas; y nivel de competencias 4: profesionales, científicos e intelectuales, y miembros del poder ejecutivo, legislativo, personal directivo de administración pública y empresas.

7 El nivel de competencias 1, correspondiente al desempeño de tareas simples y rutinarias, requiere del ciclo de educación básica completo; el nivel de competencias 2 incluye tareas de operación de maquinaria y/o conducción de vehículos, requiriendo el ciclo de educación secundaria completo, y alguna formación técnico profesional; en el nivel de competencias 3 se encuentran ocupaciones en las que se desempeñan tareas técnicas y prácticas complejas, que requieren de un elevado conocimiento de letras y matemáticas, y se requiere nivel educacional superior; por último, el nivel de competencias 4, que abarca tareas que exigen solución de problemas complejos y adopción de decisiones, requiere enseñanza superior de 3 a 6 años. 
Como es posible apreciar en el Gráfico 3, los chilenos y el total de extranjeros residentes en el país tienen una distribución similar de ocupaciones según el nivel de competencias requerido, pero los tres grupos analizados tienen distribuciones muy dispares. Los venezolanos destacan por el elevado porcentaje de ellos que tienen ocupaciones que requieren un nivel de competencias 4 (47\%) mientras que entre los colombianos predominan quienes tienen ocupaciones con nivel de competencias 2 (59\%). Finalmente, más del 90\% del colectivo haitiano se emplea en ocupaciones poco calificadas.

En el Gráfico 4 se muestra el tipo de trabajo en cuanto a nivel de competencias que desempeñan los colectivos estudiados, según los años de escolaridad; de esta forma es posible aproximar si los migrantes calificados están empleados en actividades acorde a su calificación o si se encuentran desempeñando labores de baja calificación. $\mathrm{El}$ análisis muestra que en términos generales existe correspondencia entre nivel de estudios y las competencias de las ocupaciones de las poblaciones estudiadas; sin embargo, se aprecia también que un grupo de la población calificada no logra obtener empleos acordes a su nivel de instrucción. Esta brecha es lo que se conoce como "sobrecalificación de la mano de obra”, y sería más grande para venezolanos, haitianos y colombianos que para el total de extranjeros (Gil Alonso y Domingo, 2008). El hecho de que sean poblaciones que, en promedio, han llegado a Chile en tiempos muy recientes, podría explicar la mayor sobrecalificación relativa de estos colectivos. En todo caso, la segmentación del mercado laboral que se manifiesta en el análisis no se produce sólo en función del país de origen, sino que además se relaciona con el nivel de instrucción. Esta brecha de sobrecalificación de la mano de obra es más grande para los grupos de origen haitiano y venezolano, por su mayor porcentaje de empleo en ocupaciones poco calificadas, los primeros, y por su mayor nivel medio de formación, los segundos. 
GRÁFICO 4

NIVEL EDUCACIONAL Y CATEGORÍA OCUPACIONAL SEGÚN NIVEL DE COMPETENCIAS POR POBLACIONES, OCUPADOS ENTRE 15 Y 64 AÑOS

(Distribución porcentual según años de escolaridad)

A. Chile

Nivel 1 Nivel 2 Nivel 3 Nivel 4

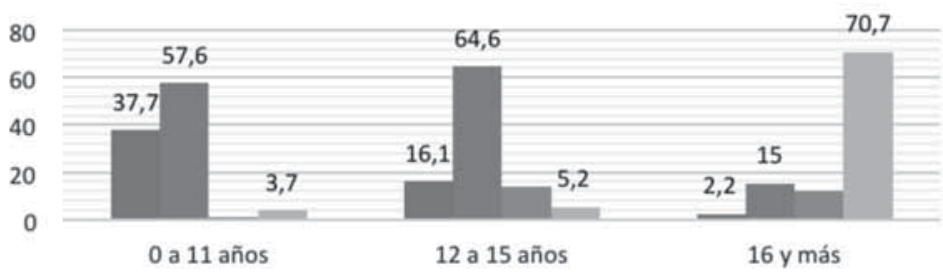

B. Extranjeros

nivel 1 Nivel 2 Nivel 3 Nivel 4

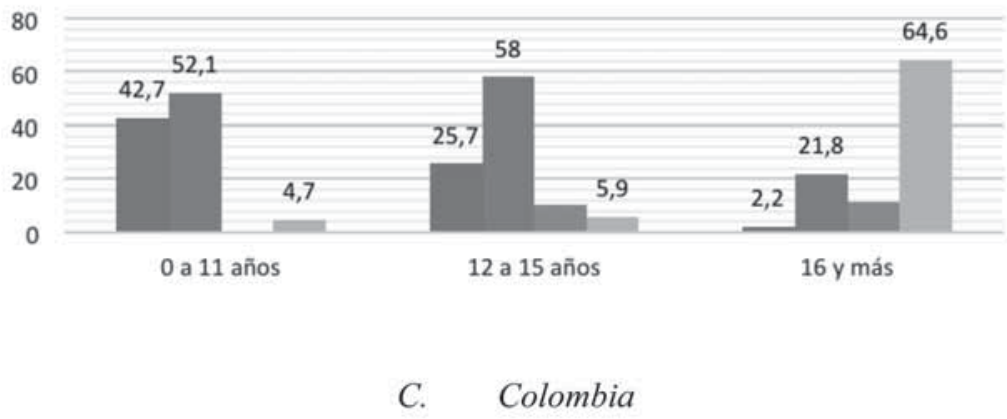

nivel 1 Nivel 2 Nivel 3 Nivel 4

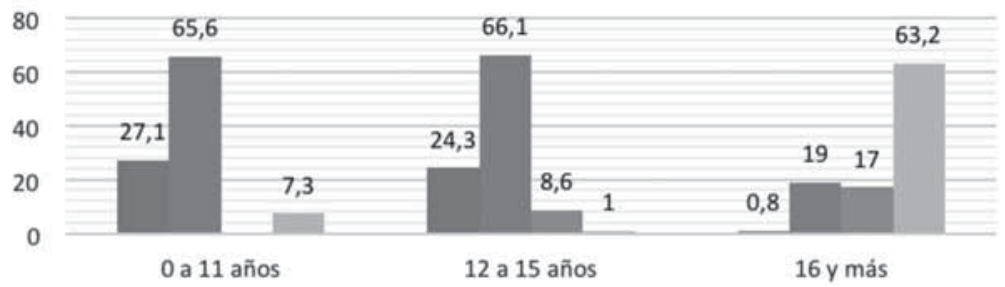




\section{Venezuela}

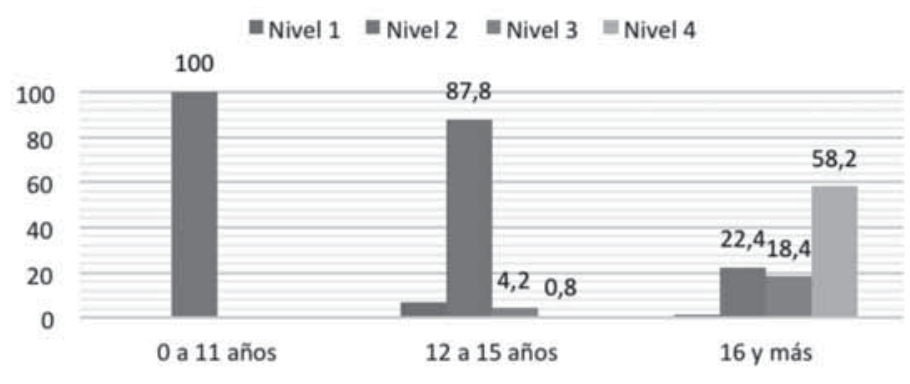

\section{E. Haiti}

Nivel 1 Nivel 2 Nivel $3=$ Nivel 4

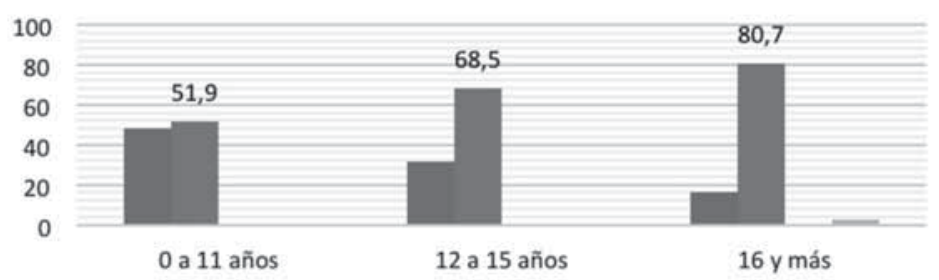

Fuente: elaboración propia con microdatos Ministerio de Desarrollo Social (2017).

\section{CONCLUSIONES Y DISCUSIÓN}

La inmigración de colombianos, venezolanos y haitianos en Chile ha aumentado exponencialmente entre los años 2002 y 2015, transformando el patrón tradicional de migración que había predominado hasta ahora, en el que la principal inmigración provenía desde países fronterizos y de ultramar. La inmigración a Chile es ahora más numerosa, diversa y compleja que unas décadas atrás, generando nuevos retos respecto a la integración y el bienestar de unos colectivos cada vez más heterogéneos. Los grupos analizados en este artículo son una muestra de dicha diversidad, pues si los tres tienen la característica común de haberse establecido en el país de manera mayoritaria en los últimos años, se diferencian en la práctica 
totalidad de sus características socioeconómicas, representando un interesante estudio de caso para analizar las implicaciones que ello tiene para su integración en el mercado laboral y, consecuentemente, en sus niveles de pobreza y vulnerabilidad social.

Desde un punto de vista puramente demográfico, la población venezolana y colombiana sigue la tendencia de la feminización de las migraciones que se ha observado en Latinoamérica y a nivel mundial. Por el contrario, la inmigración haitiana en Chile es mayoritariamente masculina, quebrando el patrón de feminización de las migraciones latinoamericanas y acercándose a otros modelos geográficos y culturales, como el de la migración de origen africano, mayoritariamente masculina (Martínez y Orrego, 2016).

Las condiciones socioeconómicas de venezolanos, colombianos y haitianos asentados en Chile son heterogéneas entre sí y también en comparación con chilenos y el total de extranjeros. El colectivo venezolano es el que presenta condiciones de asentamiento más favorables, con indicadores de nivel educacional, pobreza e ingreso mejores que chilenos y la totalidad de extranjeros residentes en el país; su mayor problema de integración en el mercado laboral chileno es el de sobrecalificación de su mano de obra, pues una parte no despreciable de los venezolanos no encuentran empleos acordes a su nivel educativo. Por su parte, el colectivo colombiano es el más importante de los tres y el que presenta mayor diversidad interna; aunque como grupo no presenta en principio una gran desventaja en cuanto a nivel educacional, sus datos respecto al nivel de competencias de las ocupaciones desempeñadas por los trabajadores colombianos y sus resultados en lo que respecta a los indicadores de pobreza multidimensional, demuestran que una parte significativa de esta población se está enfrentando a importantes situaciones de vulnerabilidad social y riesgo de exclusión. Sin embargo, peor es la situación a la que se enfrentan los haitianos residentes en el país: el nivel educacional, más bajo que el de las demás poblaciones, los sitúa de inmediato en una condición desfavorable en el momento de intentar entrar en el mercado de trabajo, lo que se traduce en el bajo nivel de competencias de sus ocupaciones, su menor ingreso medio y su mayor nivel de pobreza, tanto si se mide por ingreso como con el indicador multidimensional. Así, los haitianos residentes en el país son el colectivo más pobre, más vulnerable y con condiciones más desfavorables en relación con cualquiera de los otros grupos de análisis, mientras que los venezolanos se encuentran en la situación opuesta. 
No obstante, los resultados han mostrado que, si bien los colombianos y los venezolanos tienen un nivel educacional y calificación igual y/o superior a la población chilena, sin embargo, su acceso al mercado de trabajo no se hace sin problemas, y frecuentemente no han podido obtener - al menos hasta la fecha- empleos acordes a su nivel de formación. Además, aunque la mayoría de ellos trabajan con contrato (incluso en proporción superior a la de los chilenos, en el caso de los venezolanos) y, por lo tanto, se presupone que su estancia en Chile es legal, sin embargo, su acceso al sistema de salud y de protección social es mucho más bajo que la población chilena. Tanto este problema como el de la sobrecalificación de la mano de obra podría ser debido al establecimiento reciente en el país de estos colectivos, pero el hecho que otros grupos de extranjeros que llevan más años de residencia en Chile presenten problemas similares nos lleva a preguntarnos si las dificultades de inserción laboral de los extranjeros, en comparación con los chilenos, no se deberían exclusivamente a las diferencias socioeconómicas de partida de las nacionalidad analizadas (por ejemplo, el nivel educativo), sino al propio hecho de ser inmigrante. Ello nos hace preguntarnos si la condición migratoria no se estará transformando en un eje de desigualdad social en Chile.

Los resultados verifican, pues, la validez de la hipótesis de partida, dado que los desiguales niveles de vulnerabilidad y riesgo de exclusión social entre los tres colectivos analizados -y, en general, entre las diferentes nacionalidades-derivan de una inserción desigual en el mercado de trabajo chileno que, en parte, está a su vez condicionada por las características socioeconómicas de partida, fundamentalmente el capital formativo de los inmigrantes. Pero no sólo eso, las desigualdades que afectan a todos los colectivos, incluso a los mejor situados - como los venezolanos- muestran que debe haber otros factores que intervienen para forzar su mayor vulnerabilidad. Nos referimos, siguiendo a otros autores, a la mirada que sobre los distintos grupos de inmigrantes tiene la población chilena, que se transforma en comportamientos más o menos extendidos de racismo y discriminación que afectan sobre todo a los inmigrantes fenotípicamente "menos europeos" y, especialmente, a los afrodescendientes; sería el caso de la población haitiana —que además no es hispanohablante-, seguramente la más pobre, vulnerable y discriminada.

Los resultados creemos que han validado también el uso de la encuesta CASEN para realizar estudios sobre la inmigración en Chile, siempre que la muestra recoja suficientemente los colectivos 
analizados y que se tengan en cuenta ciertos sesgos y salvedades metodológicas. En efecto, este análisis establece una primera aproximación a tres colectivos de la inmigración reciente en Chile, pero se trata de datos que no proceden de un censo exhaustivo sino de una encuesta que, aunque tenga una amplia muestra, sólo accede a una parte de la población, la que es más accesible y la que está más dispuesta a responder a los encuestadores. Por lo tanto, la encuesta CASEN puede introducir un sesgo a favor de los hogares y personas con domicilio estable y mejor situados en el mercado laboral. En este sentido, la situación descrita por CASEN 2015 respecto a los colectivos analizados nos muestra una realidad, en relación a los aspectos socioeconómicos y laborales, que es probablemente más optimista que la verdaderamente existente, especialmente entre los sectores inmigrantes más vulnerables.

Por todo ello, y teniendo en cuenta las distintas características de los colectivos de inmigrantes de acuerdo con su país de origen, los resultados de este artículo sugieren la necesidad de profundizar las investigaciones en cada uno de ellos por separado, y de avanzar hacia estudios cuantitativos (basados en los datos del Censo de 2017, en la explotación de registros administrativos y en la mejora de las encuestas existentes y en el lanzamientos de otras nuevas) y cualitativos (observación participante, entrevistas en profundidad, grupos de discusión, entre otras), que permitan obtener conocimientos más profundos sobre la conformación de redes sociales y de prácticas transnacionales, y sobre los problemas de adaptación y discriminación de los inmigrantes extranjeros en Chile.

\section{AGRADECIMIENTOS}

Este trabajo fue financiado por CONICYT, Programa de Formación de Capital Humano Avanzado, DOCTORADO BECAS CHILE 2015 y es también un resultado de los proyectos I+D+i CSO201565219-C2-1-R y RTI2018-095667-B-I00, dirigidos por los doctores Isabel Pujadas, Fernando Gil y Cristina López, y financiados por la Agencia Estatal de Investigación y el Fondo Europeo de Desarrollo Regional (AEI/FEDER, UE). Los doctores Fernando Gil e Isabel Pujadas pertenecen al grupo de investigación consolidado Territorio, Población y Ciudadanía (GRC_2017SGR1298). 


\section{REFERENCIAS}

Allen, A. J., y Fazito, D. (2017). Mecanismos de selectividad y destinos principales de emigrantes argentinos y venezolanos: un análisis comparado. Notas de Población, 105, 191-219.

Arango, J. (2000). Explaining migration: a critical view. International Social Science journal, 52(165), 283-296.

Arango, J. (2003). La explicación teórica de las migraciones: luz y sombra. Migración y desarrollo, 1(1), 1-30.

Cerrutti, M., y Parrado, E. (2015). Intraregional Migration in South America: Trends and a Research Agenda. Annual Review of Sociology, 41, 399-421.

Czaika, M., y Haas, H. (2015). The globalization of migration: Has the world become more migratory? International Migration Review, 48(2), 283-323.

Díaz, C. (2017). Migración internacional, envejecimiento poblacional y segunda transición demográfica, ¿Hacia dónde va Chile? Notas de Población, 105, 221-257.

Durand, J., y Massey, D. (2010). New world orders: Continuities and changes in Latin American migration. The Annals of the American Academy of Political and Social Science, 630(1), 20-52.

Gil-Alonso, F. (2010). Análisis de dos propuestas metodológicas para estimar las salidas de extranjeros de España: las bajas por caducidad padronales y la renovación de las tarjetas de residencia temporales. Estadistica Española, 52(174), 277-309.

Gil-Alonso, F., y Domingo, A. (2008). Latinoamericanos en el mercado de trabajo español, 2000-2005. Papeles de Población, 14(55), 145-172.

Gil-Alonso, F., y Vidal-Coso, E. (2015). Inmigrantes extranjeros en el mercado de trabajo español: ¿más resilientes o más vulnerables al impacto de la crisis?, Migraciones, 37, 97-123.

Giorguli, S. E., y Angoa, M. A. (2016). International migration, gender and family: A miroir from Latin America. En M. J. White (Ed.), International Handbook of Migration and Population Distribution. Dordrecht: Springer.

Gissi-Barbieri, E. N., y Ghio-Suárez, G. (2017). Integración y exclusión de inmigrantes colombianos recientes en Santiago de Chile: estrato socioeconómico y "raza” en la geocultura del sistema-mundo. Papeles de Población, 23(93), 151-179.

INE (2014). Auditoría técnica a la base de datos del levantamiento censal año 2012 (24 de septiembre de 2014). Santiago de Chile: Instituto Nacional de Estadísticas.

Martínez, J., y Orrego, C. (2016). Nuevas tendencias y dinámicas migratorias en América Latina y el Caribe. Serie Población y Desarrollo, 116, Santiago de Chile: CEPAL CEPAL.

Martínez, J., Cano V., y Soffia, M. (2014). Tendencias y Patrones de la Migración latinoamericana y caribeña hacia 2010 y desafíos para una agenda regional, Serie Población y Desarrollo, 109. Santiago de Chile: CEPAL. 
Martínez, J. (Ed). (2011). Migración internacional en América Latina y el Caribe. Nuevas tendencias, nuevos enfoques. Santiago de Chile: CEPAL.

Martínez, J. (Ed.). (2008). América Latina y el Caribe: migración internacional, derechos humanos y desarrollo. Santiago de Chile: CEPAL.

Martínez, J. (2005). Magnitud y Dinámica de la Inmigración en Chile, según el Censo de 2002. Papeles de Población, 44, 109-147.

Martínez, J. (2004). El encanto de los datos: Sociodemografía de la inmigración en Chile según el censo de 2002. Serie Población y Desarrollo, 49, Santiago de Chile: CEPAL.

Massey, D. S., Arango, J., Hugo, G., Kouaouci, A., Pellegrino, A., y Taylor, J. E. (2000). Teorías sobre la migración internacional: una reseña y una evaluación. Trabajo, 2(3), 5-50.

Massey, D. S., Arango, J., Hugo, G., Kouaouci, A., Pellegrino, A., y Taylor, J. E. (1993). Theories of international migration: A review and appraisal. Population and Development Review, 431-466.

Massey, D. S. (1988). Economic development and international migration in comparative perspective. Population and Development Review, 383-413.

Massey, D. S., y García, F. (1987). The social process of international migration. Science, 237(4816), 733-738.

NACIONES UNIDAS-OIT (2017). Coyuntura Laboral en América Latina y el Caribe. La inmigración laboral en América Latina. (Informe Núm. 16). Santiago de Chile.

OBIMID (2016). La Migración en Chile: Breve Reporte y Caracterización. Madrid: Observatorio Iberoamericano sobre Movilidad Humana, Migraciones y Desarrollo.

Piore, M. J. (1979). Birds of passage: migrant labor and industrial societies. Cambridge: Cambridge University Press.

Rojas, N., Amode, N., y Vásquez, J. (2015). Racismo y matrices de "inclusión” de la migración haitiana en Chile: elementos conceptuales y contextuales para la discusión. Polis. Revista Latinoamericana, 2015(42).

Soto-Alvarado, S., (2018). La inmigración internacional reciente en Chile. El proceso migratorio en la región de Los Lagos (Tesis doctoral). Universidad de Barcelona, España.

Stefoni, C. (2005). Migración en Chile. Revista Colección Ideas, 6(59).

Tijoux, M. E. (2016). Racismo en Chile: la piel como marca de la inmigración. Santiago: Editorial Universitaria de Chile.

Tijoux, M. E., y Córdova, M. G. (2015). Racismo en Chile: colonialismo, nacionalismo, capitalismo. Polis. Revista Latinoamericana, 2015(42).

Valenzuela, P., Riveros, K., Palomo, N., Araya, I., Campos, B., Salazar, C., y Tavie, C. (2014). Integración laboral de los inmigrantes haitianos, dominicanos y colombianos en Santiago de Chile. Antropologías del Sur, 1(2).

Vega, D. (2017). Migración y recesión económica: tendencias recientes en el saldo y los flujos de migrantes latinoamericanos y caribeños en España. Notas de Población. 
Villares, M., y Oso, L. (2005). Mujeres inmigrantes latinoamericanas y empresariado étnico: dominicanas en Madrid, argentinas y venezolanas en Galicia. Revista galega de economía, 14(1-2), 261-278. 